\title{
NOUVELLE
}

\section{Des bactéries intestinales responsables des maladies nutritionnelles du foie éradiquées par phagothérapie} Une nouvelle cible thérapeutique

Dragos Ciocan ${ }^{1,2}$, Anne-Marie Cassard ${ }^{1}$
La MAF est la principale cause de cirrhose en France, où elle représente un problème majeur de santé publique. Sa forme la plus grave, l'hépatite alcoolique sévère, est associée à un risque élevé de mortalité. La maladie est traitée avec des corticostéroïdes, mais avec une efficacité limitée. La transplantation hépatique est le seul traitement curatif, mais elle n'est accessible qu'à un nombre limité de patients. Pour la NAFLD, malgré de nombreux essais cliniques effectués ces derniers années, les options thérapeutiques sont également limitées, et la transplantation hépatique reste la meilleure option pour les formes avancées [1].

\author{
${ }^{1}$ Université Paris-Saclay, \\ Inserm, Inflammation, microbiome et \\ immunosurveillance, 92140 Clamart, France. \\ ${ }^{2} \mathrm{AP}$-HP, Service d'hépato-gastroentérologie \\ et nutrition, Hôpital Antoine-Béclère, \\ 157 rue de la Porte de Trivaux, 92140 Clamart, \\ France. \\ ciocanelro@yahoo.com \\ cassard.doulcier@u-psud.fr
}

Cependant, ces maladies nutritionnelles hépatiques ne se développent que chez certains patients, ce qui pourrait être expliqué par un polymorphisme génétique. La dernière décennie a permis de montrer que le microbiote ${ }^{l}$ intestinal était également un acteur clé dans la susceptibilité $(\rightarrow)$ Voirle numéro individuelle à ces thématique Le micromaladies $(\rightarrow)$. biote : cet inconnu qui réside en nous, $\mathrm{m} / \mathrm{s}$ $n^{\circ} 11$, novembre 2016, pages $999-1016$

\footnotetext{
${ }^{1}$ Le microbiote désigne l'ensemble des micro-organismes bactéries, microchampignons, protistes, virus - vivant dans un écosystème donné. Chez l'homme, les études portent principalement sur les microbiotes cutané, vaginal, buccal et surtout, intestinal.
} 


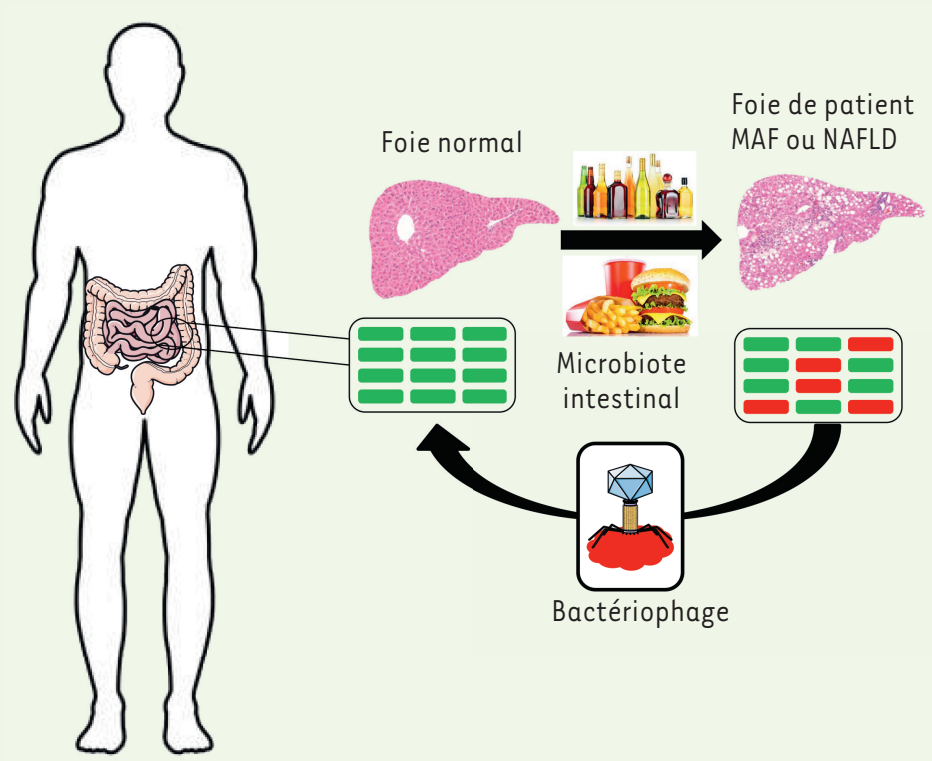

Figure 1. La phagothérapie pour éradiquer des bactéries intestinales impliquées dans les maladies nutritionnelles hépatiques. La maladie alcoolique du foie (MAF) et la non alcoholic fatty liver disease (NAFLD) présentent la même évolution physiopathologique. Enterococcus faecalis et une souche de Klebsiella pneumoniae productrice d'alcool, surabondantes (rectangles rouges) dans le microbiote intestinal de patients, ont été identifiées comme contribuant aux lésions du foie, respectivement dans la MAF et la NAFDL [7,8]. En effet, dans les deux cas, l'éradication ciblée de la bactérie intestinale par phagothérapie a permis de prévenir les lésions hépatiques dans des modèles murins.

$\varepsilon n$ effet, les patients ayant une MAF ou une NAFLD présentent des modifications spécifiques de leur microbiote en fonction du stade de la maladie [2,3], et nous avons montré que le transfert d'un microbiote intestinal humain spécifique de ces maladies à des souris suffisait à leur transmettre une susceptibilité individuelle à ces maladies $[4,5]$.

Dans ce contexte, plusieurs pistes ont été envisagées pour améliorer le contrôle de ces deux maladies en utilisant comme cible le microbiote intestinal.

1. La transplantation fécale, qui est associée à un rapport bénéfice-risque incertain lié au choix du donneur.

2. L'identification des bactéries protectrices spécifiques de la MAF ou de la NAFLD, ou d'un microbiote protecteur qui pourrait être administré aux patients comme le serait un probiotique.

3. L'identification des bactéries nocives et leur éradication. Des essais cliniques

utilisant des traitements antibiotiques sont en cours, mais cette approche manque de spécificité et comporte le risque d'induire des résistances bactériennes aux antibiotiques. Un ciblage spécifique des bactéries délétères apparaît donc nettement préférable. Les bactériophages sont des virus qui infectent les bactéries avec une spécificité liée à l'espèce bactérienne, offrant ainsi un ciblage spécifique [6]. Leur utilisation thérapeutique, envisagée depuis longtemps [1113] $(\rightarrow)$ pourrait se révéler pertinente contre la MAF et la NAFLD, comme l'indiquent

$(\rightarrow)$ Voir la Chronique génomique de $B$. Jordan, $\mathrm{m} / \mathrm{s} \mathrm{n}^{\circ} 10$, octobre 2019 , page 806 , la Nouvelle de L. Chaffraingeon et al., $m / s n^{\circ} 6-7$, juin-juillet 2019 , page 581 , et la Synthèse de N. Dufour et L. Debarbieux, $m / s n^{\circ} 4$, avril 2017, page 410

deux articles récents qui en ont testé l'efficacité dans des modèles murins $[7,8]$.
En 2017, le groupe dirigé par B. Schnabl (San Diego, États-Unis) avait montré que les patients alcooliques qui consomment des antiacides ont un risque accru de développer des formes sévères de MAF. Ces patients présentaient une augmentation de la proportion d'Enterococcus faecalis au sein de leur microbiote intestinal, et l'administration d' $\varepsilon$. faecalis à des souris suffisait à aggraver les lésions hépatiques consécutives à une alcoolisation [9]. En 2019, le même groupe a montré que si cette bactérie commensale représente moins de $0,1 \%$ des bactéries intestinales chez des individus sains, elle est 3000 fois plus abondante chez des individus souffrant d'une hépatite alcoolique [7]. $\varepsilon$. faecalis est une espèce capable de produire une cytolysine, une bactériocine $^{2}$ responsable de la lyse de bactéries à Gram positif, mais pouvant également induire la lyse de cellules de l'épithélium intestinal. De fait, la présence de cytolysine dans les fèces est corrélée à la mortalité chez les patients ayant une hépatite alcoolique sévère. Afin de démontrer le potentiel thérapeutique de l'éradication de cette bactérie, des souris alcoolisées (correspondant à un modèle murin de MAF) ont été traitées soit avec une souche $d^{\prime} \varepsilon$. faecalis produisant la cytolysine, soit avec une souche ne produisant pas de cytolysine. Les souris recevant la souche d'E. faecalis produisant la cytolysine ont présenté des lésions hépatiques plus importantes, qu'un traitement par un bactériophage ciblant $\varepsilon$. faecalis a permis de faire régresser. Ces résultats mettent en évidence le potentiel thérapeutique de la phagothérapie. Néanmoins, si $\varepsilon$. faecalis est surabondante chez $80 \%$ des patients ayant une hépatite alcoolique, elle ne produit la cytolysine que chez $30 \%$ d'entre eux. D'autres bactéries qui pourraient participer à la susceptibilité individuelle du développement d'une

\footnotetext{
2 Les bactériocines sont des peptides ou des protéines synthétisés naturellement par certaines bactéries et possédant des propriétés antibiotiques.
} 
MAF et qui pourraient être la cible d'une phagothérapie restent vraisemblablement à identifier.

De fait, les résultats récents obtenus en étudiant une bactérie particulière au cours de la NAFLD renforcent cette hypothèse [8]. En 2013, il avait été montré, dans une cohorte d'adolescents obèses, que les patients ayant une NAFLD/NASH présentaient un microbiote intestinal différent de celui des patients obèses sans NAFLD/NASH. On observait notamment un enrichissement en protéobactéries, un phylum qui comprend une grande diversité d'espèces bactériennes, dont certaines produisent de l'alcool. Effectivement, les concentrations plasmatiques d'alcool étaient plus élevées chez ces patients obèses ayant une NAFLD/NASH, sans qu'une relation de cause à effet ait pu être établie avec la maladie [10]. Grâce à l'étude publiée en 2019 par Yuan et ses collègues, la relation de causalité entre une bactérie productrice d'alcool et la NAFLD est désormais démontrée [8]. L'identification d'une telle bactérie a été faite chez un patient ayant une NASH et présentant un syndrome «d'auto-brasserie». Ce syndrome extrêmement rare avait précédemment été associé à la sur-représentation d'une levure du genre Saccharomyces. Chez ce patient, le microbiote intestinal présentait une proportion élevée de protéobactéries associée à une concentration plasmatique d'alcool élevée [8]. Parmi les espèces bactériennes surabondantes chez ce patient, les auteurs ont montré que la proportion de Klebsiella pneumoniae, une espèce bactérienne dont différentes souches sont capables de produire de l'alcool (High-alcohol-producing strains of Klebsiella pneumoniae, HiAlc$\mathrm{Kpn}$ ), était 800 fois plus élevée que chez les individus sains. De plus, dans une cohorte de patients ayant une NAFLD pour laquelle la maladie hépatique était améliorée par une perte de poids, l'amélioration de la NAFLD a été corrélée à une diminution de la présence de cette espèce bactérienne. Afin de prouver le rôle de cette bactérie dans les lésions hépatiques de NAFLD, deux souches de HiAlc-Kpn ont été isolées du patient atteint du syndrome d'auto-brasserie. La bactérie a été administrée à des souris, qui ont alors développé des lésions hépatiques similaires à celles de souris ayant reçu de l'alcool, prouvant ainsi le rôle causal des souches HiAlc-Kpn dans la NAFLD, et suggérant l'intérêt de supprimer cette bactérie de l'intestin des patients. Des phages ciblant les bactéries HiAlc-Kpn ont donc été administrés aux souris ayant reçu soit les bactéries elles-mêmes, soit une transplantation fécale contenant ces bactéries. L'élimination de ces bactéries par le bactériophage a été étroitement corrélée à une prévention des lésions hépatiques chez les souris traitées. L'éradication de ces bactéries chez des patients ayant une NAFLD/NASH pourrait donc constituer une nouvelle piste de traitement de cette maladie. Néanmoins, il s'avère qu'en dehors du syndrome d'auto-brasserie, la concentration plasmatique d'alcool chez des patients NAFLD et NASH porteurs de ces souches bactériennes est très faible et difficilement quantifiable. Les auteurs ont toutefois démontré qu'un test de stimulation orale au glucose, en fournissant aux bactéries HiAlc-Kpn le substrat pour la synthèse d'alcool, permet d'augmenter le taux d'alcool plasmatique jusqu'à des valeurs mesurables. Ce test peut donc être utilisé pour identifier les patients porteurs de bactéries $K$. pneumoniae productrices d'alcool.

Ces deux études montrent qu'au-delà de la dysbiose ${ }^{3}$ intestinale observée chez les patients atteints de MAF ou de NAFDL/ $\mathrm{NASH}$, des bactéries bien définies, telles que $\varepsilon$. faecalis ou $K$. pneumoniae, participent à l'apparition des lésions hépatiques. Mais la présence d' $\varepsilon$. faecalis ou K. pneumoniae n'est pas retrouvée chez tous les patients, et d'autres bactéries jouant un rôle délétère restent donc sans doute à identifier. La possibilité

${ }^{3}$ La dysbiose désigne le déséquilibre d'un microbiote entraînant une rupture de la symbiose entre l'hôte et son microbiote. d'éradiquer ces bactéries avec des bactériophages ouvre un nouveau champ de thérapies ciblées dans ces deux maladies hépatiques. $\diamond$

Intestinal bacteria involved in nutritionall liver disease killed by phagotherapy: a new therapeutic target

\section{LIENS D'INTÉRÊT}

Les auteurs déclarent n'avoir aucun lien d'intérêt concernant les données publiées dans cet article.

\section{RÉFÉRENCES}

1. Friedman SL, Neuschwander-Tetri BA, Rinella M, Sanyal AJ. Mechanisms of NAFLD development and therapeutic strategies. Nat Med 2018 ; 24 : 908-22.

2. Ciocan D, Voican CS, Wrzosek L, et al. Bile acid homeostasis and intestinal dysbiosis in alcoholic hepatitis. Aliment Pharmacol Ther 2018 ; 48 : 961-74.

3. Loomba R, Seguritan V, Li W, et al. Gut microbiomebased metagenomic signature for non-invasive detection of advanced fibrosis in human nonalcoholic fatty liver disease. Cell Metab 2017 ; 25 : 1054-62 el055.

4. Llopis M, Cassard AM, Wrzosek L, et al. Intestinal microbiota contributes to individual susceptibility to alcoholic liver disease. Gut $2016 ; 65: 830-9$.

5. Le Roy T, Llopis M, Lepage P, et al. Intestinal microbiota determines development of non-alcoholic fatty liver disease in mice. Gut $2013 ; 62$ : 1787-94.

6. Mirzaei MK, Maurice CF. Menage a trois in the human gut: interactions between host, bacteria and phages. Nat Rev Microbiol 2017 ; 15 : 397-408.

7. Duan $Y$, Llorente C, Lang $S$, et al. Bacteriophage targeting of gut bacterium attenuates alcoholic liver disease. Nature 2019 ; 575 : 505-11.

8. Yuan J, Chen C, Cui J, et al. Fatty liver disease caused by high-alcohol-producing Klebsiella pneumoniae. Cell Metab 2019 ; 30 : 675-88 e677.

9. Llorente C, Jepsen P, Inamine T, et al. Gastric acid suppression promotes alcoholic liver disease by inducing overgrowth of intestinal Enterococcus. Nat Commun $2017 ; 8: 837$.

10. Zhu L, Baker SS, Gill C, et al. Characterization of gut microbiomes in nonalcoholic steatohepatitis (NASH) patients: a connection between endogenous alcohol and NASH. Hepatology $2013 ; 57: 601-9$.

11. Jordan B. Cent ans après, le retour de la phagothérapie? Med Sci (Paris) 2019 ; 35 : 806-9.

12. Chaffringeon L, De La Cruz J, Dettling V, et al. Les bactériophages, alliés de l'épithélium intestinal contre les bactéries pathogènes. Med Sci (Paris) 2019 ; 35 : 581-3.

13. Dufour N, Debarbieux L. La phagothérapie : une arme crédible face à l'antibiorésistance. Med Sci (Paris) $2017 ; 33: 410-6$.

14. Gilgenkrantz H, Teillaud JL (coordinateurs). Le microbiote : cet inconnu qui réside en nous. Med Sci (Paris) $2016 ; 32: 919-1016$.

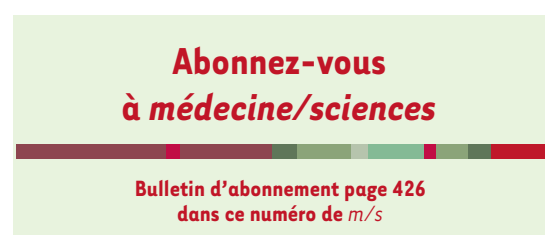

